\title{
Signature RNAS and related regulatory roles in type 1 diabetes mellitus based on competing endogenous RNA regulatory network analysis
}

Qinghong Shi ${ }^{1}$ and Hanxin $\mathrm{YaO}^{2^{*} \text { (D) }}$

\begin{abstract}
Background: Our study aimed to investigate signature RNAs and their potential roles in type 1 diabetes mellitus (T1DM) using a competing endogenous RNA regulatory network analysis.

Methods: Expression profiles of GSE55100, deposited from peripheral blood mononuclear cells of 12T1DM patients and 10 normal controls, were downloaded from the Gene Expression Omnibus to uncover differentially expressed long non-coding RNAs (IncRNAs), mRNAs, and microRNAs (miRNAs). The ceRNA regulatory network was constructed, then functional and pathway enrichment analysis was conducted. AT1DM-related ceRNA regulatory network was established based on the Human microRNA Disease Database to carry out pathway enrichment analysis. Meanwhile, the T1DM-related pathways were retrieved from the Comparative Toxicogenomics Database (CTD).

Results: In total, 847 mRNAs, 41 IncRNAs, and 38 miRNAs were significantly differentially expressed. The ceRNA regulatory network consisted of 12 IncRNAs, 10 miRNAs, and 24 mRNAs. Two miRNAs (hsa-miR-181a and hsa-miR-1275) were screened as T1DM-related miRNAs to build the T1DM-related ceRNA regulatory network, in which genes were considerably enriched in seven pathways. Moreover, three overlapping pathways, including the phosphatidylinositol signaling system (involving PIP4K2A, INPP4A, PIP4K2C, and CALM1); dopaminergic synapse (involving CALM1 and PPP2R5C); and the insulin signaling pathway (involving CBLB and CALM1) were revealed by comparing with T1DMrelated pathways in the CTD, which involved four IncRNAs (LINC01278, TRG-AS1, MIAT, and GAS5-AS1).
\end{abstract}

Conclusion: The identified signature RNAs may serve as important regulators in the pathogenesis of T1DM.

Keywords: T1DM, LnCRNAs, CeRNAs

\section{Background}

Recently, type 1 diabetes mellitus (T1DM) is a multifactorial autoimmune disease characterized by insulin deficiency and hyperglycaemia, which is considered to involve the selective attack of insulin-producing pancreatic $\beta$ cells by activated $\mathrm{T}$ lymphocytes that recognize their autoantigens [1]. T1DM accounts for approximately $5-10 \%$ of all cases of diabetes mellitus, and the incidence

\footnotetext{
*Correspondence: yhx0002001@jlu.edu.cn

${ }^{2}$ Department of Clinical Laboratory, The First Hospital of Jilin University, No. 1, Xinmin Street, Chaoyang District, Changchun 130021, Jilin, China Full list of author information is available at the end of the article
}

of T1DM is rising worldwide, with more than $80 \%$ of diabetes occurring in younger children $[2,3]$.

Many efforts have been made recently to gain insights into the pathogenesis of T1DM. Three main regions on chromosomes, including the protein tyrosine-phosphatase non-receptor-type 22 region on chromosome $1 \mathrm{p} 13$, the human leukocyte antigen region on chromosome $6 \mathrm{p} 21$, and the insulin region on chromosome $11 \mathrm{p} 15$, play essential roles in insulin expression, immune response, and $\beta$-cell function, which are associated with T1DM $[4,5]$. More than 50 genomic risk loci have been identified for T1DM based on genome-wide association 
studies [5]. However, most of the risk loci are located in non-coding genomic regions, and an increasing number of studies have focused on the potential roles of long non-coding RNAs (lncRNAs) in pancreatic islets and the pathogenesis of T1DM [6,7]. Motterle et al. found that some lncRNAs are modulated by proinflammatory cytokines during the development of T1DM in non-obese diabetic mice, which probably contributes to the sensitization of $\beta$ cells to apoptosis and failure during the initial phases of T1DM [7]. The nuclear-enriched $\beta$-cell lncRNA PLUTO may regulate the expression of $P D X 1$, which is a key pancreatic $\beta$-cell transcription factor; furthermore, knockdown of PLUTO is associated with the downregulation of $P D X 1$ in EndoC- $\beta \mathrm{H} 1$ cells and primary islet cells, implicating the roles of lncRNAs in the regulation of $\beta$-cell-specific transcription factors [8]. Therefore, examining the ability of lncRNAs to regulate gene expression and cell-specific transcription factors opens avenues to a better understanding of T1DM pathogenesis.

On the other hand, an increasing body of evidence indicates that microRNAs (miRNAs) play important roles in processes involved in the pathogenesis of T1DM, including immune system functions and $\beta$-cell metabolism and death $[9,10]$. Assmann et al. have suggested that 11 circulating miRNAs $(m i R-21-5 p, m i R-24-3 p, m i R-100-5 p$, $m i R-146 a-5 p, m i R-148 a-3 p, m i R-150-5 p, m i R-181 a-5 p$, $m i R-210-5 p$, $m i R-342-3 p$, $m i R-375$, and $m i R-1275)$ are consistently dysregulated in T1DM patients [11]. It has been further revealed that five miRNAs (miR-103a-3p, $m i R-155-5 p, \quad m i R-200 a-3 p, \quad m i R-146 a-5 p$, and $m i R-$ $210-3 p)$, which have been confirmed as dysregulated miRNAs based on plasma miRNA expression profiles of T1DM patients and control individuals, could regulate genes involved in the innate immune system-, MAPK-, apoptosis-, insulin-, and cancer-related pathways [12]. Additionally, it is widely acknowledged that competing endogenous RNAs (ceRNAs) can interact with mRNAs by competing with miRNAs, and miRNA-mediated interactions between lncRNAs and mRNAs occur in the progression of various diseases [13-15]. However, few current studies have reported on the ceRNA-based regulatory mechanisms of T1DM. In the study of Yang et al. [16] global miRNA and mRNA expressions were profiled in peripheral blood mononuclear cells from 12 patients with newly diagnosed T1DM and 10 normal controls, while miRNA-mediated interactions between lncRNAs and mRNAs were not revealed. Thus, further studies aimed at clarifying ceRNA-based transcriptional signatures are needed to provide new insights into the pathogenesis of T1DM. In our present study, differentially expressed lncRNAs, mRNAs, and miRNAs between T1DM and normal controls were identified based on profiles retrieved from the Gene Expression Omnibus [17]. Subsequently, ceRNA-based transcriptional signatures were revealed via the construction of a T1DMrelated ceRNA regulatory network.

\section{Methods \\ Data source and annotation}

Expression and non-coding RNA profiles (GEO accession number: GSE55100) deposited by Yang et al. [16] were downloaded from the National Center for Biotechnology Information GEO (https://www.ncbi.nlm.nih.gov/ geo/) [18], which consist of two subseries, GSE55098 and GSE55099. The GSE55098 contains expression data of peripheral blood mononuclear cells from 12 patients with T1DM and 10 normal control subjects, which were based on the GPL570 [HG-U133_Plus_2] Affymetrix Human Genome U133 Plus 2.0 Array platform. The GSE55099 contains microRNA expression data from peripheral blood mononuclear cells from the same 12 patients with T1DM and 10 normal control subjects, based on the GPL8786 [miRNA-1] Affymetrix Multispecies miRNA-1 Array platform. The miRNAs, lncRNAs, and mRNAs in the downloaded profiles were annotated via the Human Genome Organization Gene Nomenclature Committee (http://www.genenames.org/) [19], where over 40,000 approved gene symbols have been recorded, of which more than 19,000 are for protein-coding genes.

\section{Identification of differentially expressed RNAs}

The differentially expressed RNAs (DERs) between T1DM and normal controls were screened with Limma (Linear Models for Microarray Data) package (Version 3.34.0; https://bioconductor.org/packages/release/bioc/ html/limma.html) [20] of R3.4.1. The cut-off criteria were set as false discovery rate (FDR) less than 0.05 and $\mid \log _{2}$ fold change| greater than 0.5 . Two-way hierarchical clustering analysis based on Euclidean distance was executed for all the identified DERs via the pheatmap (Version 1.0.8, https://cran.r-project.org/web/packages/ pheatmap/index.html) of R3.4.1 [21-23]. Meanwhile, gene ontology (GO) functional enrichment in terms of biological process as well as Kyoto Encyclopedia of Genes and Genomes (KEGG) pathway enrichment analysis were conducted for differentially expressed mRNAs via the Database for Annotation, Visualization and Integrated Discovery (DAVID, Version 6.8, https://david.ncifcrf. gov/) [24, 25], with a threshold of $p<0.05$.

\section{Construction of ceRNA regulatory network}

The regulatory interactions between differentially expressed lncRNAs and miRNAs were retrieved from the DIANA-LncBase (Version 2, http://carolina.imis. athena-innovation.gr/diana_tools/web/index.php) [17]. 
The negative regulatory interactions with a miRNA target gene score (miTG-score) larger than 0.6 (the default threshold in DIANA-LncBase) were retained to construct the lncRNA-miRNA regulatory network, which was visualized with Cytoscape (Version 3.6.1, https:// cytoscape.org/) [26].

The target genes of differentially expressed miRNAs were obtained by using starBase (Version 2.0, http:// starbase.sysu.edu.cn/), which contains prediction data from the five databases of targetScan, picTar, RNA22, PITA, and miRanda [25]. The negative regulatory interactions predicted in at least one of the five databases were retained to construct the miRNA-mRNA regulatory network, which was visualized with Cytoscape (Version 3.6.1, https://cytoscape.org/) [26].

Afterwards, the IncRNA-miRNA-mRNA (1) regulatory network was constructed by integrating the above lncRNA-miRNA regulatory interactions with the miRNA-mRNA regulatory interactions, and visualized with Cytoscape (Version 3.6.1, https://cytoscape.org/) [26]. Moreover, GO functional enrichment in terms of biological process and pathway enrichment analysis were conducted for genes in the ceRNA regulatory network via DAVID (Version 6.8, https://david.ncifc rf.gov/) $[24,25]$, and $p<0.05$ was used as the cut-off criterion.

\section{T1DM-related ceRNA regulatory network}

The miRNAs associated with T1DM were obtained by using the Human microRNA Disease Database (HMDD; Version 3.2, http://www.cuilab.cn/hmdd) [27], with the option "Disease name" filled with "type 1 diabetes mellitus." The disease-related miRNAs were mapped onto the ceRNA regulatory network to reveal the target genes for pathway enrichment analysis via DAVID (Version 6.8, https://david.ncifcrf.gov/) [24, 25] and $p<0.05$ was used as the cut-off criterion. In addition, the sequences of interested IncRNAs and miRNAs were downloaded from Ensembl genome browser (http://asia.ensembl.org/index.html) and miRBase (http://www.mirbase.org/), respectively. Binding sites between lncRNAs and miRNAs were predicted by using miRanda (http://cbio.mskcc.org/miRNA2003/ miranda.html) [28], which is an algorithm for finding genomic targets for microRNAs.

On the other hand, the KEGG pathways associated with T1DM were screened from the Comparative Toxicogenomics Database update 2019 (http://ctd.mdibl.org/) [29], with the keyword "type 1 diabetes mellitus." The enriched KEGG pathways from DAVID were compared with those obtained from the Comparative Toxicogenomics Database (CTD).

\section{Results}

\section{Data annotation and DER screening}

According to the platform annotation information, 946 lncRNAs, 597 miRNAs, and 10,085 mRNAs were received. By comparing the sample profiles of patients with T1DM with those of normal controls, 926 DERs were identified using Limma package, including 847 mRNAs (448 downregulated and 399 upregulated), 41 lncRNAs (22 downregulated and 19 upregulated), and 38 miRNAs (18 downregulated and 20 upregulated; Fig. 1). Moreover, the hierarchical clustering analysis revealed that all mRNAs and lncRNAs identified could adequately distinguish between T1DM samples and normal samples (Fig. 1a). Meanwhile, T1DM samples could also be discriminated from normal samples based on the expression levels of the 38 differentially expressed miRNAs (Fig. 1b).

The GO functional enrichment analysis indicated that the above differentially expressed mRNAs were significantly associated with the biological processes of cellular defense response $(p=3.020 \mathrm{E}-07)$, immune response $(p=4.320 \mathrm{E}-07)$, regulation of immune response $(p=2.730 \mathrm{E}-06)$, innate immune response $(p=4.610 \mathrm{E}-06)$, cell surface receptor signaling pathway $(p=8.640 \mathrm{E}-06)$, adaptive immune response $(p=2.170 \mathrm{E}-05)$, and inflammatory response $(p=5.040 \mathrm{E}-05$; Additional file 1: Table S1, Fig. 2a). At the same time, differentially expressed mRNAs were enriched in 18 KEGG pathways, such as osteoclast differentiation $(p=4.930 \mathrm{E}-04)$, natural-killer-cell-mediated cytotoxicity $(p=6.850 \mathrm{E}-04)$, antigen processing and presentation $(p=1.008 \mathrm{E}-02)$, cytokine-cytokine receptor interaction $(p=1.250 \mathrm{E}-02)$, and glycerophospholipid metabolism ( $p=1.520 \mathrm{E}-02$; Additional file 1: Table S1, Fig. 2b).

\section{Constructed ceRNA regulatory network}

The regulatory interactions between differentially expressed lncRNAs and differentially expressed miRNAs were acquired by using the DIANA-LncBase version 2 database. The 94 pairs of negative lncRNA-miRNA regulatory interactions with a coefficient larger than 0.6 were retained to build the IncRNA-miRNA regulatory network, which consisted of 61 nodes, 36 miRNAs, and 25 lncRNAs (Additional file 2: Figure S1). The target genes of differentially expressed miRNAs were predicted using starBase. Afterwards, the 915 pairs of negative miRNAmRNA regulatory interactions involving differentially expressed mRNAs were submitted to construct the miRNA-mRNA regulatory network, which consisted of 322 nodes, 23 miRNAs, and 299 mRNAs (Additional file 3: Figure S2).

By integrating the lncRNA-miRNA regulatory interactions with miRNA-mRNA regulatory interactions, 


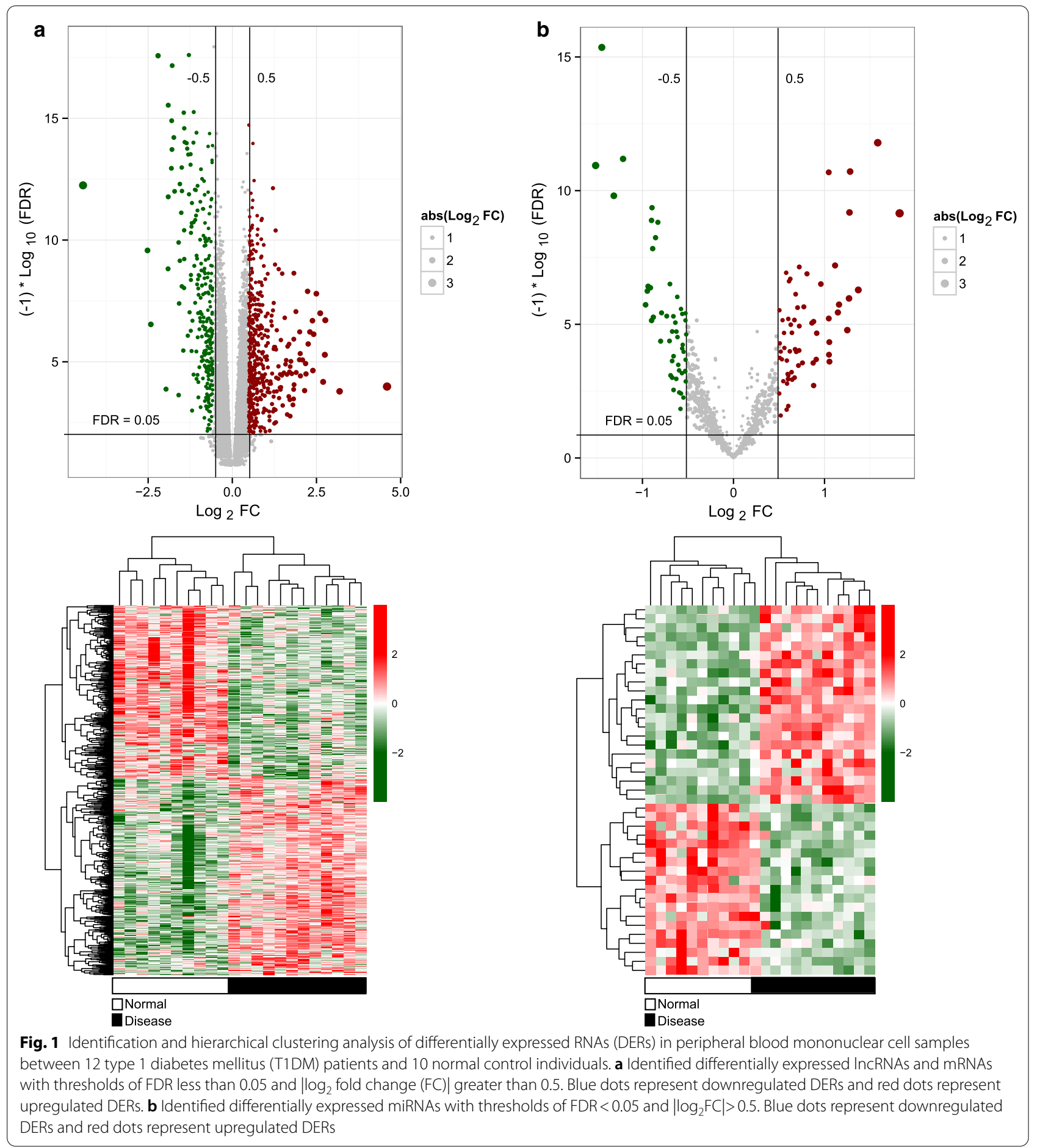

the lncRNA-miRNA-mRNA (1) regulatory network was established (Fig. 3). There were 360 nodes and 1,002 regulatory interactions in the ceRNA regulatory network, including 12 lncRNAs, 10 miRNAs, and 24 mRNAs. The mRNAs in the ceRNA regulatory network were substantially related to $14 \mathrm{GO}$ biological processes, such as cell maturation $(p=2.434 \mathrm{E}-03)$, negative regulation of transcription, DNA templated $(p=2.598 \mathrm{E}-03)$, negative regulation of fat cell differentiation $(p=4.295 \mathrm{E}-03)$, and small GTPase-mediated signal transduction $(p=5.980 \mathrm{E}-03$; Fig. $4 \mathrm{a}$, Additional file 4: Table S2). Meanwhile, eight KEGG 
a

GO:0071222 cellular response to lipopolysaccharide -

GO:0050852 T cell receptor signaling pathway -

GO:0050776 regulation of immune response -

GO:0050728 negative regulation of inflammatory response -

GO:0045766 positive regulation of angiogenesis -

GO:0045087 innate immune response -

GO:0043065 positive regulation of apoptotic process GO:0035556 intracellular signal transduction -

GO:0032496 response to lipopolysaccharide -

GO:0032088 negative regulation of NF-kappaB transcription factor activity GO:0031295 T cell costimulation GO:0030168 platelet activation -

$\stackrel{\xi}{\varrho}$

GO:0019221 cytokine-mediated signaling pathway GO:0009615 response to virus GO:0008360 regulation of cell shape GO:0007596 blood coagulation -

GO:0007264 small GTPase mediated signal transduction GO:0007204 positive regulation of cytosolic calcium ion concentration -

GO:0007166 cell surface receptor signaling pathway GO:0007165 signal transduction GO:0006968 cellular defense response GO:0006955 immune response GO:0006954 inflammatory response GO:0006915 apoptotic process GO:0002250 adaptive immune response GO:0001934 positive regulation of protein phosphorylation -

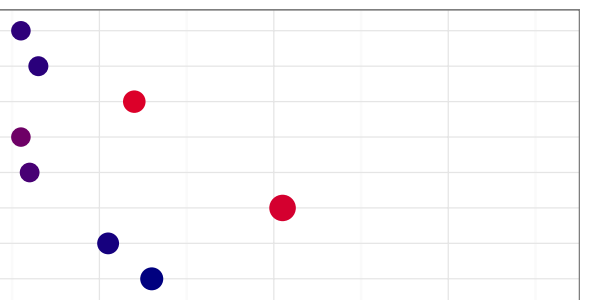

- $\log ($ PValue, 10)
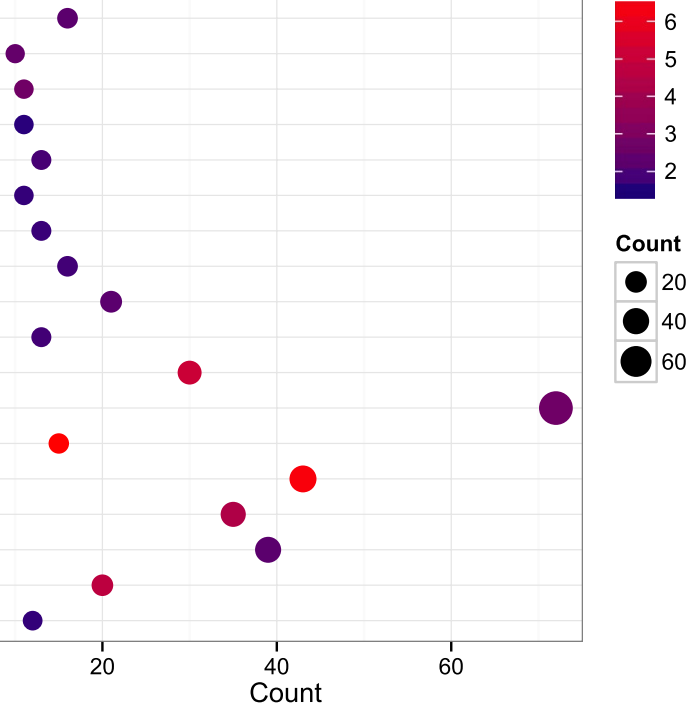

$$
\text { Count }
$$

b

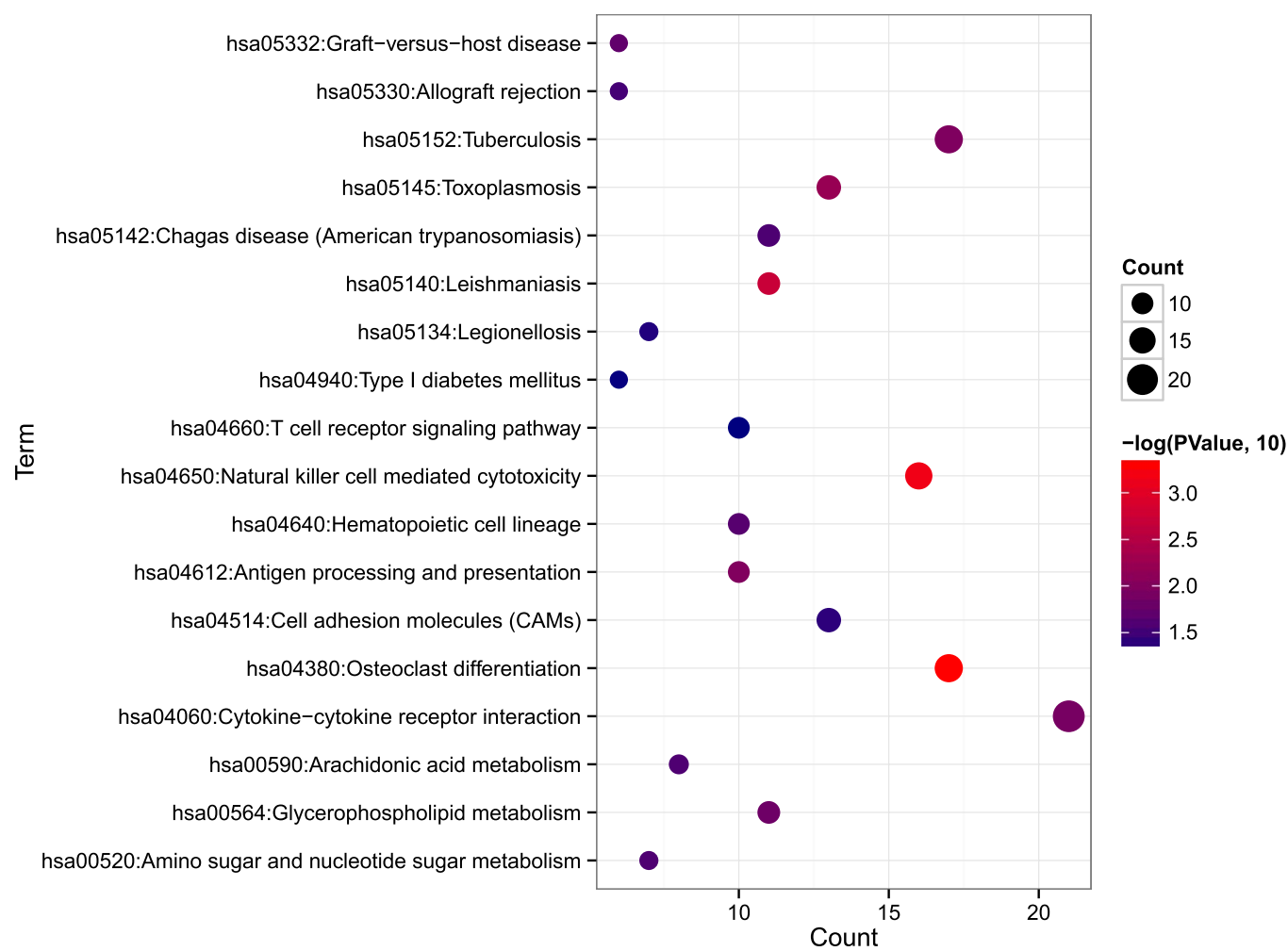

Fig. 2 Gene ontology (GO) functional enrichment in terms of biological process and Kyoto Encyclopedia of Genes and Genomes (KEGG) pathway enrichment analysis for differentially expressed mRNAs. a Enriched GO terms for differentially expressed mRNAs. b Enriched KEGG pathways for differentially expressed mRNAs 


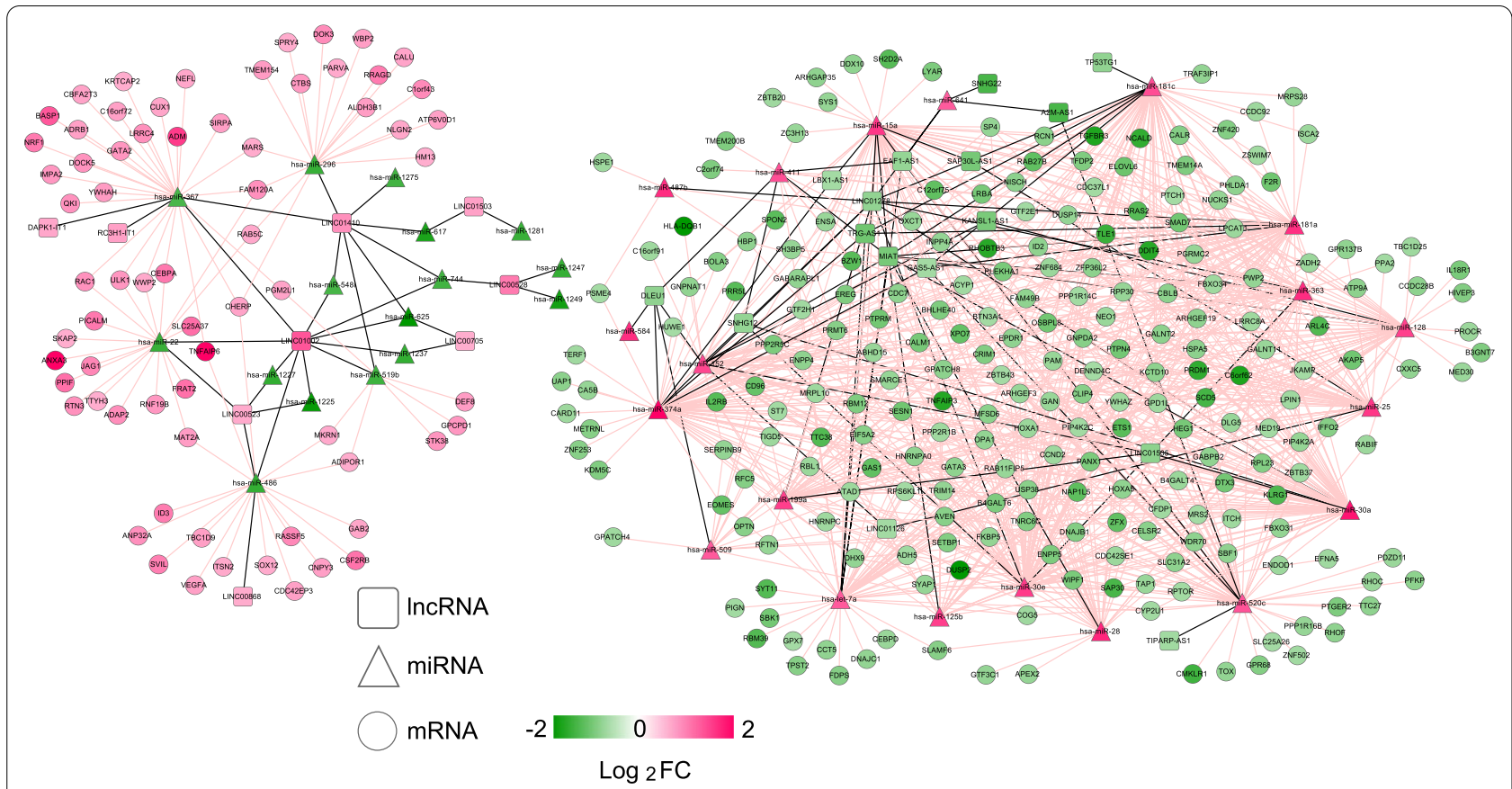

Fig. 3 The constructed competing endogenous RNA (1) regulatory network. Square, triangle, and circle nodes represent IncRNAs, miRNAs, and mRNAs, respectively. Nodecolors range from green to red, which indicate downregulated to upregulated expression changes
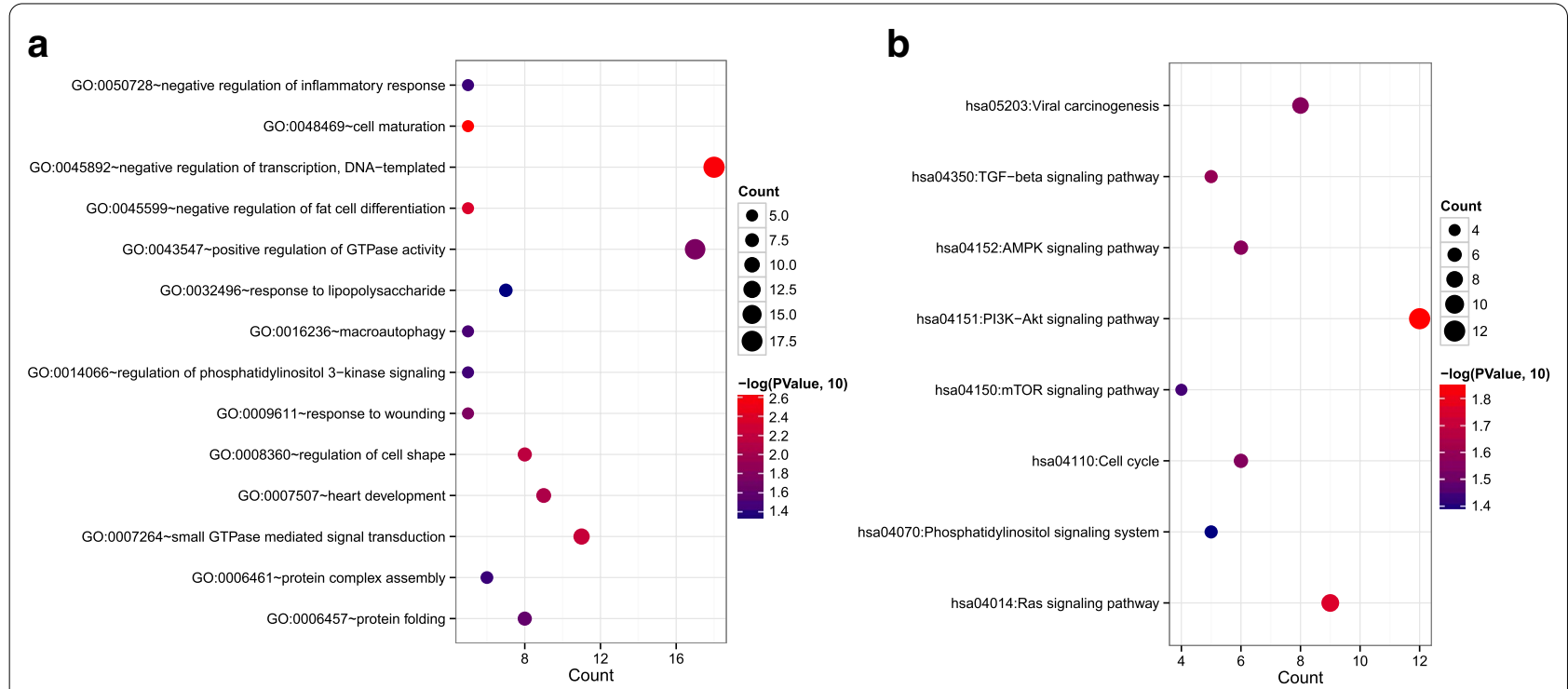

Fig. 4 GO functional enrichment in terms of biological process and KEGG pathway enrichment analysis for mRNAs in the ceRNA regulatory network. a Enriched GO terms for mRNAs in the ceRNA regulatory network. $\mathbf{b}$ Enriched KEGG pathways for mRNAs in the ceRNA regulatory network

pathways were enriched for the mRNAs in the ceRNA regulatory network, including the PI3K-Akt signaling pathway $(p=1.442 \mathrm{E}-02)$, the Ras signaling pathway $(p=1.753 \mathrm{E}-02)$, and the TGF-beta signaling pathway $(p=2.579 \mathrm{E}-02$; Fig. 4b, Additional file 4: Table S2).

\section{T1DM-related ceRNA regulatory network}

The miRNAs related to T1DM were downloaded from HMDD Version 3.2 with the disease name "type 1 diabetes mellitus," and 32 miRNAs were obtained. By comparing with the miRNAs in the ceRNA regulatory 
network, two overlapping miRNAs (hsa-miR-181a and hsa-miR-1275) were retained, and the regulatory network involving these two miRNAs was separated from the ceRNA regulatory network (Fig. 5a). The genes in the separated regulatory network were significantly enriched in seven pathways, including the phosphatidylinositol signaling system $(p=4.567 \mathrm{E}-04)$, inositol phosphate metabolism $(p=2.504 \mathrm{E}-03)$, mucin type O-Glycan biosynthesis $(p=1.029 \mathrm{E}-02)$, the ErbB signaling pathway $(p=2.636 \mathrm{E}-02)$, glycerophospholipid metabolism $(p=2.842 \mathrm{E}-02)$, dopaminergic synapse $(p=3.634 \mathrm{E}-02)$, and the insulin signaling pathway $(p=3.856 \mathrm{E}-02$; Table 1). Binding sites of hsa-miR-181a with GAS5-AS1, LINC01278, and MIAT were exhibited in Additional file 5: Figure S3. The possible binding sites of $h s a-$ miR-1275 with LINC01410 were shown in the Additional file 6: Figure S4.

The KEGG pathways related to T1DM were further searched in the CTD with the keyword "type 1 diabetes mellitus," and 148 KEGG pathways were revealed. Three overlapping KEGG pathways were revealed by comparing these 148 KEGG pathways with the above seven enriched pathways, including the phosphatidylinositol signaling system, dopaminergic synapse, and the insulin signaling pathway. The disease pathway network showed that four downregulated lncRNAs (LINC01278, TRG-AS1, $M I A T$, and GAS5-AS1) could regulate overexpressed hsa-miR-181a, which had six downregulated target genes that were involved in the three KEGG pathways (Fig. $5 \mathrm{~b}$ ). PIP4K2A, INPP4A, PIP4K2C, and CALM1 were involved in the phosphatidylinositol signaling system. $C B L B$ and $C A L M 1$ were related to the insulin signaling pathway. In the dopaminergic synapse, CALM1 and PPP2R5C were enriched.

\section{Discussion}

Selective destruction of insulin-producing pancreatic islet $\beta$ cells causes T1DM, a chronic immune-mediated and inflammatory disease [30]. In recent years, increasing efforts have been made to understand the molecular mechanisms of the pathogenesis of T1DM; and, researching ceRNA regulatory network-based RNA signatures may contribute to gaining further insights into its specific regulatory roles in T1DM. In our study, 926

Table 1. Seven significantly enriched Kyoto Encyclopedia of Genes and Genomes (KEGG) pathways for mRNAs in type 1 diabetes mellitus (T1DM) related competing endogenous (ceRNA) regulatory network

\begin{tabular}{llll}
\hline Term & Count & P value & Genes \\
\hline *hsa04070:Phosphatidylinositol signaling system & 4 & $4.567 \mathrm{E}-04$ & INPP4A, PIP4K2A, PIP4K2C, CALM1 \\
hsa00562:Inositol phosphate metabolism & 3 & $2.504 \mathrm{E}-03$ & INPP4A, PIP4K2A, PIP4K2C \\
hsa00512:Mucin type O-Glycan biosynthesis & 2 & $1.029 \mathrm{E}-02$ & GALNT2, GALNT11 \\
hsa04012:ErbB signaling pathway & 2 & $2.636 \mathrm{E}-02$ & CBLB, EREG \\
hsa00564:Glycerophospholipid metabolism & 2 & $2.842 \mathrm{E}-02$ & GPD1L, LPCAT1 \\
*hsa04728:Dopaminergic synapse & 2 & $3.634 \mathrm{E}-02$ & PPP2R5C, CALM1 \\
*hsa04910:Insulin signaling pathway & 2 & $3.856 \mathrm{E}-02$ & CBLB, CALM1 \\
\hline
\end{tabular}

*The pathway overlapping with T1DM-related pathways retrieved from Comparative Toxicogenomics Database (CTD)
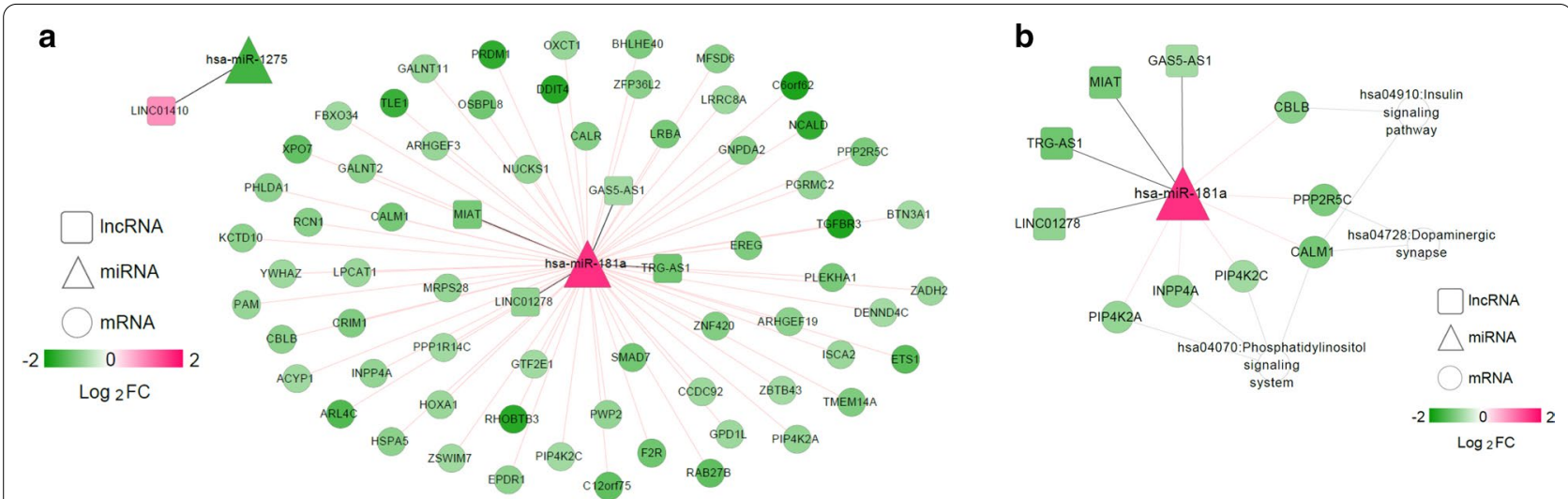

Fig. 5 T1DM-related ceRNA regulatory network and disease pathway network. a The T1DM-related ceRNA regulatory network. b The disease pathway network. Square, triangle, and circle nodes represent IncRNAs, miRNAs, and mRNAs, respectively. Nodecolors range from green to red, which indicate downregulated to upregulated expression changes 
DERs were identified in expression profiles of peripheral blood mononuclear cells from T1DM patients, including 847 mRNAs, 41 lncRNAs, and 38 miRNAs. In the study of Yang et al. [16] 24 miRNAs and 1218 genes were found differently expressed in patients with newly diagnosed T1DM. Expression levels of hsa-let-7a, hsamiR-1275, hsa-miR-22, hsa-miR-25, hsa-miR-28, and $h s a-m i R-486$, which were identified as differentially expressed miRNAs by Yang et al., were also found significantly different in our study. Liu et al. [31] have downloaded GSE55100 and differentially expressed miRNA-mRNA interactions were unveiled from the 7 miRNAs (hsa-miR-374a, hsa-miR-146b, hsa-miR-181a, hsa-miR-19b, hsa-miR-125b, hsa-let-7f, and hsa-miR28) and 651 mRNAs. In our study, differentially expressed miRNA-mRNA regulatory interactions involved 23 miRNAs and 299 mRNAs, and 4 miRNAs (hsa-miR374a, hsa-miR-181a, hsa-miR-125b, and hsa-miR28) were consistent with the previous study. In order to revel potential hub genes involved in the pathogenesis of Chinese type 1 diabetic patients, GSE55100 has been also downloaded to identify DEGs, and thirteen hub genes (MMP9, ARG1, CAMP, CHI3L1, CRISP3, SLPI, LCN2, PGLYRP1, LTF, RETN, CEACAM1, CEACAM8, and MS4A3) were retrieved by module analysis [32]. These thirteen hub genes were also identified as differentially expressed genes in our study.

Besides, two miRNAs (hsa-miR-181a and hsamiR-1275) were screened as T1DM-related miRNAs based on information from the HMDD to construct the T1DM-related ceRNA regulatory network. The genes in the T1DM-related ceRNA regulatory network were significantly enriched in seven pathways, and three overlapping pathways, including the phosphatidylinositol signaling system, dopaminergic synapse, and the insulin signaling pathway,were revealed by comparing with T1DM-related pathways in the CTD. The disease pathway network contained one upregulated miRNA ( $h s a-$ miR-181a) and four lncRNAs (LINC01278, TRG-AS1, MIAT, and GAS5-AS1). Overexpression of miR-181a levels were found in insulin-resistant cultured hepatocytes, and inhibition of miR-181a may lead to increased protein levels of SIRT1, which is a potential therapeutic target for combating insulin resistance, and thereby improving hepatic insulin sensitivity [33]. It is well known that hyperglycaemia, acidosis, and insulin resistance play significant roles in T1DM [34]. In a study by Nielsen et al., 12 upregulated miRNAs, including miR-152, miR30a-5p, miR-181a, miR-24, miR-148a, miR-210, miR27a, miR-29a, miR-26a, miR-27b, miR-25, and miR-200a, were identified in T1DM patients [35]. Thus, the changes in expression of $h s a-m i R-181 a$ in this study were consistent with the results of previous studies.
Moreover, six target genes (PIP4K2A, INPP4A, PIP4K2C, CALM1, CBLB, and PPP2R5C) of hsa-miR$181 a$ were involved in a further established disease pathway network based on the T1DM-related ceRNA regulatory network. T1DM arises from autoimmunemediated $\beta$-cell destruction, and this defect is closely associated with the molecular levels found in the insulin signaling pathway [36, 37]. Casitas B-lineage lymphoma $\mathrm{b}(C B L B)$, a member of the CBL/SLI family of ubiquitin-protein ligases, functions as a key regulator of lymphocyte activation and autoimmunity [38, 39]. Komeda diabetes-prone rats, which are a spontaneous animal model of human type 1 diabetes, as well as $C B L B$-deficient mice, have been observed to have infiltrations of lymphocytes into pancreatic islets, the thyroid gland, and kidneys, suggesting that $C B L B$ dysfunction leads to autoimmune processes [40, 41]. Transgenic complementation with wild type $C B L B$ greatly suppresses the development of the Komeda diabetes-prone phenotype, indicating that $C B L B$ is a negative regulator of autoimmunity and a susceptibility gene for T1DM in the rat [42]. Moreover, one single nucleotide polymorphism in exon 12 of the $C B L B$ gene has been shown to be associated with T1DM based on analysis of a large Danish T1DM database of 480 families [43]. In our study, expression levels of $C B L B$ were also lower in the peripheral blood mononuclear cell profiles of T1DM patients. Thus, $C B L B$ is a key gene of T1DM, and downregulated $C B L B$ participating in the insulin signaling pathway may contribute to the autoimmune disease of T1DM. In addition, regulatory interactions between four IncRNAs (LINC01278, TRG-AS1, $M I A T$,and GAS5-AS1) and hsa-miR-181a were revealed in the T1DM-related ceRNA regulatory network. However, there have been no reports, to our knowledge, of the roles of LINC01278, TRG-AS1, MIAT, and GAS5-AS1 in the pathogenesis of T1DM. According to the ceRNA hypothesis, it can be surmised that these four lncRNAs (LINC01278, TRG-AS1, MIAT, and GAS5-AS1) might participate in regulating the expression levels of target genes of $h s a-m i R-181 a$ by competing with hsa-miR-181a.

\section{Conclusion}

In conclusion, 847 mRNAs, 41 lncRNAs, and 38 miRNAs were significantly differentially expressed in peripheral blood mononuclear cells of T1DM patients. A disease pathway network was made based on the T1DM-related ceRNA regulatory network, which consisted of four lncRNAs (LINC01278, TRG-AS1, MIAT, and GAS5-AS1), one miRNA (hsa-miR-181a), six target genes, as well as three important pathways related to T1DM, including the phosphatidylinositol signaling system (involving PIP4K2A, INPP4A, PIP4K2C, and CALM1), the dopaminergic synapse (involving CALM1 and PPP2R5C), 
and the insulin signaling pathway (involving $C B L B$ and $C A L M 1)$. These results suggest that the signature RNAs identified above may serve as important regulators in the pathogenesis of T1DM. However, the expression changes of miRNA and mRNAs should be further detected in different patient cohort by using laboratory experiments. More particularly, four lncRNAs, LINC01278, TRG-AS1, $M I A T$, and GAS5-AS1, may compete with hsa-miR-181 to regulate its target genes in T1DM.

\section{Abbreviations}

T1DM: Type 1 diabetes mellitus; IncRNAs: Long non-coding RNAs; miRNAs: MicroRNAs; CTD: Comparative Toxicogenomics Database; ceRNAs: Competing endogenous RNAs; DERs: Differentially expressed RNAs; GO: Gene ontology; KEGG: Kyoto Encyclopedia of Genes and Genomes.

\section{Supplementary Information}

The online version contains supplementary material available at https://doi. org/10.1186/s12920-021-00931-0.

Additional file 1: Table S1. The significantly enriched gene ontology (GO) biological processes and Kyoto Encyclopedia of Genes and Genomes (KEGG) pathways for differentially expressed mRNAs

Additional file 2: Figure $\mathbf{S 1}$. The constructed IncRNA-miRNA regulatory network. Square and triangle nodes represent IncRNAs and miRNAs, respectively. Nodecolors range from green to red, which indicate downregulated to upregulated expression changes.

Additional file 3: Figure S2. The constructed miRNA-mRNA regulatory network. Triangle and circle nodes represent miRNAs and mRNAs, respectively. Nodecolors range from green to red, which indicate downregulated to upregulated expression changes. The phosphatidylinositol signaling system, dopaminergic synapse, and the insulin signaling pathway were the three major pathways related to T1DM.

Additional file 4: Table S2. The significantly enriched gene ontology (GO) biological processes and Kyoto Encyclopedia of Genes and Genomes (KEGG) pathways for mRNAs in the competing endogenous (1) regulatory network.

Additional file 5: Figure S3. Binding sites of hsa-miR-181a with GAS5-AS1, LINC01278, and MIAT.

Additional file 6: Figure S4. Possible binding sites of hsa-miR-1275 with LINC01410.

\section{Acknowledgements}

None.

\section{Authors' contributions}

QHS and HXY participated in the design of this study, and they both performed the statistical analysis. QHS carried out the study and collected important background information. HXY drafted the manuscript. All authors read and approved the final manuscript.

\section{Funding}

None.

\section{Availability of data and materials}

GSE55100 (https://www.ncbi.nlm.nih.gov/geo/query/acc.cgi?acc=GSE55100) contains two subseries GSE55098 (https://www.ncbi.nlm.nih.gov/geo/query/ acc.cgi?acc=GSE55098)) based on the GPL570 ([HG-U133_Plus_2] Affymetrix Human Genome U133 Plus 2.0 Array) and GSE55099 (https://www.ncbi.nlm. nih.gov/geo/query/acc.cgi?acc=GSE55099) based on the GPL8786 ([miRNA-1] Affymetrix Multispecies miRNA-1 Array), were downloaded from the National Center for Biotechnology Information (NCBI) Gene Expression Omnibus (GEO, https://www.ncbi.nlm.nih.gov/geo/). The raw data were collected and analyzed by the Authors, and are not ready to share their data because the data have not been published.

\section{Declarations}

Ethics approval and consent to participate

Not applicable.

\section{Consent for publication}

Not applicable.

\section{Competing interests}

The authors declare no conflict of interest.

\section{Author details}

${ }^{1}$ Department of Clinical Laboratory, The Third Hospital of Jilin University, No. 126, Xiantai Street, Changchun 130033, Jilin, China. ${ }^{2}$ Department of Clinical Laboratory, The First Hospital of Jilin University, No. 1, Xinmin Street, Chaoyang District, Changchun 130021, Jilin, China.

Received: 30 August 2020 Accepted: 4 March 2021

Published online: 18 May 2021

\section{References}

1. Katsarou A, Gudbjörnsdottir S, Rawshani A, Dabelea D, Bonifacio E, Anderson B, et al. Type 1 diabetes mellitus. Nat Rev Dis Primers. 2017;3:17016.

2. Penn MMC. Diabetes type I. In: Parnham MJ, editor. Compendium of inflammatory diseases. Basel: Springer; 2016. p. 448-54.

3. Barnett R. Type 1 diabetes. Lancet. 2018;391(10117):30024-32.

4. Nyaga DM, Vickers MH, Jefferies C, Perry JK, O'Sullivan JM. The genetic architecture of type 1 diabetes mellitus. Mol Cell Endocrinol. 2018:477:70-80.

5. Sharp SA, Weedon MN, Hagopian WA, Oram RA. Clinical and research uses of genetic risk scores in type 1 diabetes. Curr Opin Genet Dev. 2018:50:96-102.

6. Mirza AH, Kaur S, Pociot F. Long non-coding RNAs as novel players in $\beta$ cell function and type 1 diabetes. Hum Genomics. 2017;11(1):17.

7. Motterle A, Gattesco S, Caille D, Meda P, Regazzi R. Involvement of long non-coding RNAs in beta cell failure at the onset of type 1 diabetes in NOD mice. Diabetologia. 2015;58(8):1827-35.

8. Akerman I, Beucher Z, Rolando A, Sauty-Colace DMY. Human pancreatic cell IncRNAs control cell- specific regulatory networks. Cell Metab. 2016;25(2):400-11.

9. Guay C, Roggli E, Nesca V, Jacovetti C, Regazzi R. Diabetes mellitus, a microRNA-related disease? TransI Res J Lab Clin Med. 2011;157(4):253-64.

10. Guay C, Regazzi R. Circulating microRNAs as novel biomarkers for diabetes mellitus. Nat Rev Endocrinol. 2013;9(9):513-21.

11. Assmann TS, Recamonde-Mendoza M, Souza BMD, Crispim D. MicroRNA expression profiles and type 1 diabetes mellitus: systematic review and bioinformatic analysis. Endocr Connect. 2017;6(8):EC-17-0248.

12. Assmann TS, Recamonde-Mendoza M, Puñales M, Tschiedel B, Canani LH, Crispim D. MicroRNA expression profile in plasma from type 1 diabetic patients: case-control study and bioinformatic analysis. Diabetes Res Clin Pract. 2018;141:35-46.

13. Salmena L, Poliseno L, Tay Y, Kats L, Pandolfi P. A ceRNA hypothesis: the Rosetta Stone of a hidden RNA language? Cell. 2011;146(3):353-8.

14. Huang M, Zhong Z, Lv M, Shu J, Tian Q, Chen J. Comprehensive analysis of differentially expressed profiles of IncRNAs and circRNAs with associated co-expression and ceRNA networks in bladder carcinoma. Oncotarget. 2016;7(30):47186.

15. Zhou M, Wang X, Shi H, Cheng L, Wang Z, Zhao H, et al. Characterization of long non-coding RNA-associated ceRNA network to reveal potential prognostic IncRNA biomarkers in human ovarian cancer. Oncotarget. 2016;7(11):12598.

16. Yang M, Ye L, Wang B, Gao J, Liu R, Hong J, et al. Decreased miR-146 expression in peripheral blood mononuclear cells is correlated with 
ongoing islet autoimmunity in type 1 diabetes patients 1. J Diabetes. 2015;7(2):158-65

17. Paraskevopoulou MD, Vlachos IS, Karagkouni D, Georgakilas G, Kanellos I, Vergoulis T, et al. DIANA-LncBase v2: indexing microRNA targets on noncoding transcripts. Nucleic Acids Res. 2016;44(D1):26.

18. Barrett T, Troup DB, Wilhite SE, Ledoux P, Rudnev D, Evangelista C, et al. NCBI GEO: mining tens of millions of expression profiles-database and tools update. Nucleic Acids Res. 2006;35(suppl_1):D760-5.

19. Braschi B, Denny P, Gray K, Jones T, Seal R, Tweedie S, et al. Genenames.org: the HGNC and VGNC resources in 2019. Nucleic Acids Res. 2018;47(D1):D786-92.

20. Ritchie ME, Phipson B, Wu D, Hu Y, Law CW, Shi W, et al. limma powers differential expression analyses for RNA-sequencing and microarray studies. Nucleic Acids Res. 2015;43(7):e47.

21. Szekely GJ, Rizzo ML. Hierarchical clustering via joint between-within distances: extending ward's minimum variance method. J Classif. 2005;22(2):151-83.

22. Chakerian J, Holmes S. Computational tools for evaluating phylogenetic and hierarchical clustering trees. J Comput Graph Stat. 2012;21 (3):581-99.

23. Deza MM, Deza E. Distances in graph theory. Encyclopedia of distances. Berlin: Springer; 2016. p. 277-311.

24. Da WH, Sherman BT, Lempicki RA. Systematic and integrative analysis of large gene lists using DAVID bioinformatics resources. Nat Protoc. 2008:4(1):44-57.

25. Huang DW, Sherman BT, Lempicki RA. Bioinformatics enrichment tools: paths toward the comprehensive functional analysis of large gene lists. Nucleic Acids Res. 2008;37:1-13.

26. Shannon P, Markiel A, Ozier O, Baliga NS, Wang JT, Ramage D, et al. Cytoscape: a software environment for integrated models of biomolecular interaction networks. Genome Res. 2003;13(11):2498-504.

27. Huang Z, Shi J, Gao Y, Cui C, Zhang S, Li J, et al. HMDD v3.0: a database for experimentally supported human microRNA-disease associations. Nucleic Acids Res. 2019:47(D1):D1013-7.

28. Betel D, Koppal A, Agius P, Sander C, Leslie C. Comprehensive modeling of microRNA targets predicts functional non-conserved and non-canonical sites. Genome Biol. 2010;11(8):2010-1.

29. Davis AP, Grondin CJ, Johnson RJ, Sciaky D, McMorran R, Wiegers J, et al. The comparative toxicogenomics database: update 2019. Nucleic Acids Res. 2018;47(D1):D948-54.

30. Steck AK, Rewers MJ. Genetics of type 1 diabetes. Clin Chem. 2017;57(2):176.

31. Liu S, Li H. Down-regulation of microRNA-28 in peripheral blood mononuclear cell plays a role in pathogenesis of type 1 diabetes. Int I Clin Exp Med. 2017;10:10021-30.
32. Yang $\mathrm{S}, \mathrm{Cao} \mathrm{C}$, Xie Z, Zhou Z. Analysis of potential hub genes involved in the pathogenesis of Chinese type 1 diabetic patients. Ann Transl Med. 2020;8(6):295

33. Zhou B, Li C, Qi W, Zhang Y, Zhang F, Wu JX, et al. Downregulation of miR-181a upregulates sirtuin-1 (SIRT1) and improves hepatic insulin sensitivity. Diabetologia. 2012;55(7):2032-43.

34. Ramakrishnan R. Brain signalling systems: a target for treating type I diabetes mellitus. Brain Res Bull. 2019;152:191-201.

35. Nielsen LB, Wang C, Sørensen K, Bang-Berthelsen CH, Hansen L, Andersen $M-L M$, et al. Circulating levels of microRNA from children with newly diagnosed type 1 diabetes and healthy controls: evidence that miR-25 associates to residual beta-cell function and glycaemic control during disease progression. Exp Diabetes Res. 2012;2012:896362.

36. Lake S, Krook A, Zierath JR. Analysis of insulin signaling pathways through comparative genomics. Mapping mechanisms for insulin resistance in type 2 (non-insulin-dependent) diabetes mellitus. Exp Clin Endocrinol Diabetes. 2003;111(4):191-7.

37. Hennige $A M$, Häring $H-U$. The mouse as a model of insulin signaling. Drug Discov Today Dis Models. 2004;1 (3):199-204.

38. Joazeiro CA. The tyrosine kinase negative regulator c-Cbl as a RING-type, E2-dependent ubiquitin-protein ligase. Science. 1999;286(5438):309-12.

39. Yokoi N, Fujiwara Y, Wang H-Y, Kitao M, Hayashi C, Someya T, et al. Identification and functional analysis of CBLB mutations in type 1 diabetes. Biochem Biophys Res Commun. 2008;368(1):37-42.

40. Chiang YJ, Kole HK, Brown K, Naramura M, Fukuhara S, Hu R-J, et al. $\mathrm{Cbl}-\mathrm{b}$ regulates the $\mathrm{CD} 28$ dependence of T-cell activation. Nature. 2000:403(6766):216-20.

41. Bachmaier K, Krawczyk C, Kozieradzki I, Kong Y-Y, Sasaki T, Oliveira-dosSantos A, et al. Negative regulation of lymphocyte activation and autoimmunity by the molecular adaptor Cbl-b. Nature. 2000;403(6766):211-6.

42. Yokoi N, Komeda K, Wang H-Y, Yano H, Kitada K, Saitoh Y, et al. Cblb is a major susceptibility gene for rat type 1 diabetes mellitus. Nat Genet. 2002;31(4):391-4.

43. Bergholdt R, Taxvig C, Eising S, Nerup J, Pociot F. CBLB variants in type 1 diabetes and their genetic interaction with CTLA4. J Leukoc Biol. 2005;77(4):579-85.

\section{Publisher's Note}

Springer Nature remains neutral with regard to jurisdictional claims in published maps and institutional affiliations.

Ready to submit your research? Choose BMC and benefit from:

- fast, convenient online submission

- thorough peer review by experienced researchers in your field

- rapid publication on acceptance

- support for research data, including large and complex data types

- gold Open Access which fosters wider collaboration and increased citations

- maximum visibility for your research: over $100 \mathrm{M}$ website views per year

At BMC, research is always in progress.

Learn more biomedcentral.com/submissions 